\title{
EFECTOS DEL PRESTIGIO DE LOS COLEGIOS DE ENSEÑANZA MEDIA, DE LAS UNIVERSIDADES Y EL APRENDIZAJE DEL EMPLEADOR SOBRE LOS SALARIOS EN CHILE*
}

\author{
Paola Bordón
}

\begin{abstract}
Resumen: Este estudio examina los efectos de haber asistido a un colegio privado, el ranking de universidades y el aprendizaje del empleador sobre los salarios en Chile. La estrategia empírica se basa en regresiones de salarios tipo Mincer. El análisis se realizó usando una muestra de egresados universitarios. Los principales resultados dan cuenta de un efecto importante y significativo de haber asistido a colegios privados y a universidades prestigiosas sobre los salarios de los trabajadores al inicio de su carrera laboral. Estos resultados son consistentes con un modelo de discriminación estadística y aprendizaje por parte del empleador dado que los efectos estimados disminuyen
\end{abstract}

Paola Bordón. Departamento de Economía, Universidad de Wisconsin-Madison, Madison. Correo electrónico: bordon@wisc.edu.

* Quisiera agradecer a Chao Fu, Travis Horlacher, John Kennan, Rasmus Lentz, Christopher Taber, Sergio Urzúa, a los participantes del seminario de economía laboral de la Universidad de Wisconsin-Madison, de la conferencia de LACEA Labor, y a los referentes por sus comentarios, muy útiles. También le estoy muy agradecida a Fabian Lange por compartir parte de su programa conmigo. Todos los errores son míos. 
con la experiencia laboral de los trabajadores. Utilizando este modelo, se encuentra que los empleadores reducen en un 50 por ciento la ponderación que le dan al ranking de universidades de sus trabajadores luego de tres años. Mis hallazgos, además, indican que incorporar rankings basados en el percentil de la prueba de admisión universitaria en las solicitudes de empleo puede mejorar significativamente la capacidad del mercado para asignar adecuadamente los sueldos, reduciendo la brecha informativa entre potenciales empleados y empleadores.

Palabras clave: educación superior, aprendizaje del empleador, salarios, mercado laboral.

Recibido: junio 2013; aceptado: noviembre 2013.

\section{THE EFFECTS OF PRIVATE HIGH SCHOOLS, UNIVERSITY RANKINGS AND EMPLOYER LEARNING ON WAGES IN CHILE}

Abstract: This paper studies the effects of attending a private high school, the university ranking and the employer learning on wages. The empirical strategy is based on the Mincer-type wage regressions. The analysis was carried out using individual-level data from Chile. I find a large and significant effect on wages at the beginning of workers' careers from attending a private high school and from attending a highly ranked university. These findings can be rationalized by the statistical discrimination and employer learning model since the effects of attending a private high school or highly ranked university decrease with experience. I construct an employer learning model to explain these decreases and find employers decrease by 50 per cent the weight they place on university ranking when setting wages in three years. My findings further indicate that incorporating university admission test percentile rankings in employment applications can significantly improve the market's ability to appropriately assign wages by decreasing the information gap between potential employees and employers.

Keywords: higher education, employer learning, wages, labor market.

Received: June 2013; accepted: November 2013. 


\section{Introducción}

Se cree ampliamente en Chile que en el proceso de contratación se da demasiada importancia a la escuela secundaria y a la universidad en las que estudió el postulante. Las explicaciones para esta diferencia de valoración van desde una real diferencia en la calidad de la educación, pasando por variaciones en las capacidades de acceder a redes de contacto por haber accedido a distintas instituciones hasta una discriminación estadística. La discriminación estadística se refiere al hecho de que cuando se tiene información incompleta, se espera que personas con más educación, en promedio, sean más productivas que personas con menos educación ${ }^{1}$. Por ello, las empresas utilizan variables fáciles de observar, tales como la escolaridad, para pronosticar la productividad de los trabajadores y fijar sus salarios. A medida que pasa el tiempo, las empresas aprenden la verdadera productividad de sus trabajadores, un proceso conocido como aprendizaje del empleador.

Farber y Gibbons (1996) y Altonji y Pierret (2001, en adelante AP) fueron pioneros en el estudio de la discriminación estadística y el aprendizaje del empleador. Ellos investigaron el hecho de que personas más capaces aumentan más rápidamente sus salarios con la experiencia y que el efecto de la escolaridad se reduce con el tiempo. Efectivamente, AP encuentran que los coeficientes salariales sobre las variables que afectan la productividad que no pueden ser directamente observados por las empresas, tales como su puntaje en el test de Calificación de las Fuerzas Armadas (AFQT, por sus siglas en inglés), aumentan con la experiencia, en tanto que los coeficientes de escolaridad, que las empresas pueden observar directamente, disminuyen con el tiempo. Los autores explican que estos resultados son una prueba de la discriminación estadística en base a la escolaridad.

Lange (2007) muestra cómo estimar la velocidad del aprendizaje del empleador usando mediciones observadas del rendimiento de la escolaridad y de las habilidades en distintos niveles de experiencia. Lange encuentra que los empleadores estadounidenses aprenden rápidamente. Mansour (2009) estudia aún más el aprendizaje de los empleadores

${ }^{1}$ Spence (1973) y Weiss (1995) desarrollaron modelos de señalización de habilidades donde la educación es un mecanismo que sirve para clasificar a las personas en grupos (niveles de educación) que están correlacionados con habilidades. 
usando distintas ocupaciones y Pasche (2008) incluye simultáneamente múltiples mediciones de las habilidades cognitivas y no cognitivas para probar la discriminación estadística y estimar la velocidad del aprendizaje del empleador, encontrando que los empleadores aprenden todos los tipos de habilidades de sus trabajadores, y discriminan en base a la escolaridad ${ }^{2}$.

Existen pocos papers que examinen el rendimiento de la universidad, la PSU y distintos tipos de colegios. Véase, por ejemplo, Contreras, Rodríguez y Urzúa (2013), Reyes, Rodríguez y Urzúa (2013), entre otros. Bordón y Braga (2013) prueban la hipótesis de la discriminación estadística usando el diseño de regresión discontinua, estimando un $19 \%$ de prima salarial para los recién titulados de las universidades más selectivas en Chile. Sin embargo, ellos encuentran que esta prima disminuye en tres puntos porcentuales por año de experiencia en el mercado laboral.

Este artículo estudia los efectos de asistir a un colegio secundario privado, del ranking de universidades y los del aprendizaje del empleador sobre los salarios en el mercado laboral chileno. Éste es el primer artículo, a mi entender, que amplía el modelo para incorporar características claves de los curriculum vitae chilenos: colegio y universidad a la que asistió. Los empleadores tienen esta información en los curriculum vitae de los postulantes, pero ésta, en general, no está disponible para los investigadores. Los principales resultados dan cuenta de un efecto importante y significativo en el haber asistido a colegios privados y a universidades prestigiosas sobre los salarios de los trabajadores al inicio de su carrera laboral. Estos resultados se explican por el modelo de discriminación estadística y el aprendizaje del empleador, dado que los efectos de haber asistido a colegios secundarios privados o a universidades prestigiosas disminuyen con la experiencia laboral. Se construye un modelo de aprendizaje del empleador para explicar estas disminuciones y se encuentra que los empleadores reducen en un 50 por ciento la ponderación que le dan al ranking de universidades de sus trabajadores al cabo de observar su trabajo por tres años.

Este artículo se organiza de la siguiente manera. La sección 2 describe el sistema de educación superior en Chile. La sección 3 explica

${ }^{2}$ Ver en Schönberg (2007) y Arcidiacono et al. (2010) más modelos generales de aprendizaje del empleador. 
la discriminación estadística y el aprendizaje del empleador, describe los datos y presenta los principales resultados de la estimación para el mercado laboral de egresados de universidades chilenas. La sección 4 desarrolla el modelo de aprendizaje del empleador y los resultados de la estimación. La sección 5 entrega algunas implicancias para las políticas públicas y la sección 6 entrega las principales conclusiones del artículo.

\section{Breve descripción del sistema de educación superior en Chile}

El número de alumnos matriculados en el sistema de educación superior en Chile ha aumentado significativamente desde principios de los años 1980. En 1983 había 175.250 estudiantes en el sistema, en 1990 la cantidad de alumnos matriculados había aumentado a 249.482 . El mayor crecimiento se produjo en los años 1990, cuando las matrículas se elevaron drásticamente. En 1995 las matrículas en todo el país se alzaban aproximadamente hasta los 345.000 estudiantes; en el año 2000 a 452.325 estudiantes y en 2013, las matrículas alcanzaron los 1.075.668 (véase gráfico $n^{\circ} 1$ ). Esta enorme expansión se debió a un proceso de privatización y diversificación del sistema de educación superior, destinado a generar un crecimiento económico permanente y dar estabilidad al país.

Las universidades pueden dividirse en dos categorías principales: las instituciones tradicionales y las no tradicionales. Las instituciones tradicionales incluyen a las universidades más antiguas y más prestigiosas creadas antes de 1981, y aquellas instituciones derivadas de las antiguas universidades (creadas después de 1980). Existen 25 universidades tradicionales totalmente autónomas coordinadas por el Consejo de Rectores de Universidades Chilenas (CRUCH), que pueden obtener un financiamiento parcial del Estado. Estas universidades utilizan un proceso de admisión único: la Prueba de Selección Universitaria (PSU) ${ }^{3}$. Ella a su vez está compuesta de dos pruebas obligatorias: las pruebas de lenguaje y de matemática; y pruebas opcionales: ciencias o ciencias sociales. Cada universidad fija un puntaje mínimo a ser obtenido en la PSU para ingresar y llena sus vacantes con los estudiantes que obtengan los puntajes más altos. El puntaje de corte se determina una vez finalizado el proceso de postulación y por lo tanto es incierto al momento

${ }^{3}$ En el 2004 se modificó la prueba de selección universitaria que la actualmente se llama la PSU. 
GRÁFICO N 1: $\quad$ MATRÍCULA TOTAL EN LA EDUCACIÓN SUPERIOR 1983-2013

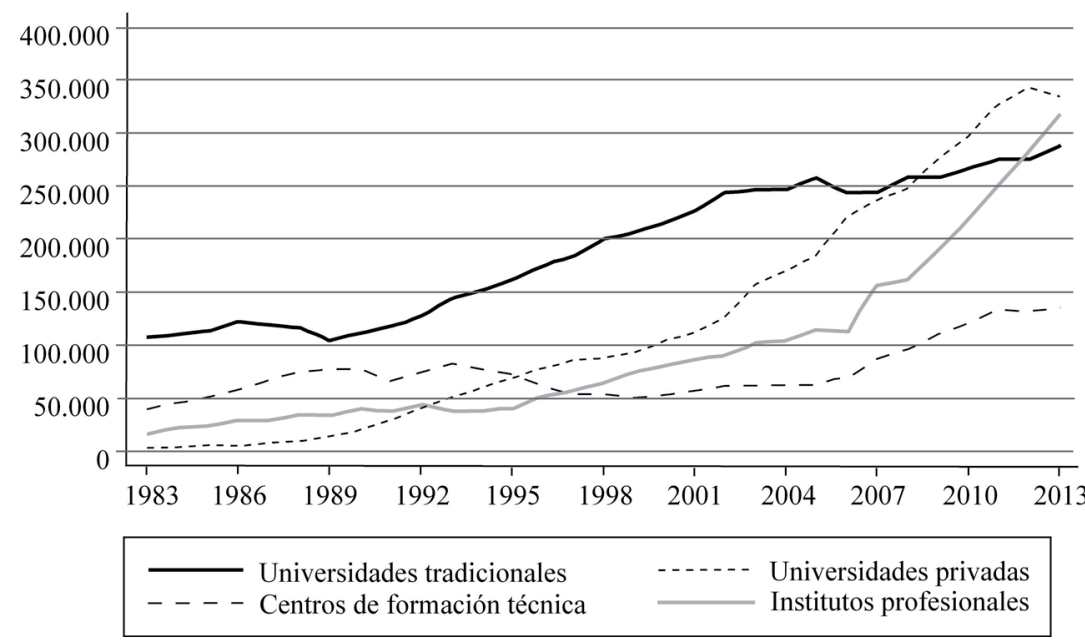

GRÁFICO N²: $\quad$ TITULADOS DE LA EDUCACIÓN SUPERIOR 1998-2011

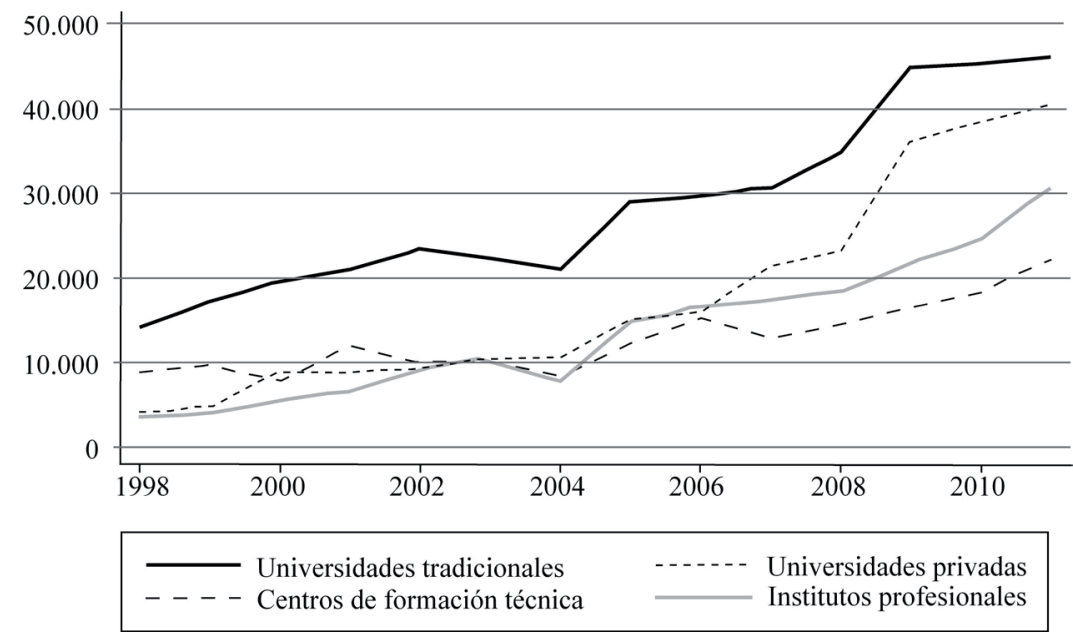


de la postulación. Los estudiantes postulan a una universidad y a una carrera simultáneamente. Después de 1981 se crearon universidades no tradicionales o privadas, que no usan necesariamente el puntaje de la PSU para seleccionar a sus estudiantes ${ }^{4}$. Todas las instituciones de educación superior cobran una matrícula y un arancel.

El aumento en la matrícula en la educación superior ha significado un mayor número de titulados durante las dos últimas décadas. En 1995, 24.400 titulados ingresaron al mercado laboral, en tanto que en el año 2000 cerca de 42.000 lo hicieron y en 2011, alrededor de 139.500. Esto significa que en quince años el número de titulados se quintuplicó (véase gráfico $\mathrm{n}^{\circ} 2$ ). Las universidades tradicionales más que cuadruplicaron el número de titulados que producen, pero las universidades privadas aumentaron 17 veces su número de los mismos. Dado que la mayoría de las universidades privadas son relativamente nuevas, es decir, que la mayoría de ellas fueron creadas a principios de los años 1990, los empleadores no saben a cabalidad cuán productivos son sus titulados en promedio. Por el contrario, los empleadores han estado contratando a trabajadores titulados de las universidades más antiguas desde hace muchos años y pueden tener una idea acerca de su productividad. Por ende, los empleadores podrían, en principio, discriminar estadísticamente a los trabajadores de universidades tradicionales, pero no podrían discriminar a trabajadores de nuevas universidades privadas.

\section{Discriminación estadística y aprendizaje del empleador}

AP (2001) desarrollaron un modelo para testear la hipótesis del aprendizaje del empleador y la discriminación estadística (EL-SD, por sus siglas en inglés). La idea principal del modelo EL-SD estriba en que los empleadores no pueden observar la productividad de sus potenciales trabajadores directamente. Por ello, los empleadores deben usar proxies de productividad fácilmente observables tales como la escolaridad y la universidad para predecir la productividad de los trabajadores. Con el tiempo, los empleadores van observando la productividad de sus em-

${ }^{4}$ A partir del proceso de admisión del año 2012, ocho universidades privadas ingresaron al sistema de admisión que utiliza la PSU para seleccionar a sus alumnos. 
pleados, proceso que se conoce como aprendizaje del empleador. Formalmente, el modelo es el siguiente:

$$
\chi_{i x}=\alpha s_{i}+\beta q_{i}+\gamma z_{i}+\eta_{i}+\mathrm{H}\left(x_{i}\right)
$$

donde $\chi_{\text {ix }}$ es el logaritmo de productividad en el mercado laboral del trabajador i con x años de experiencia. $s_{i}$ son las variables fáciles de observar, tales como la carrera y la universidad; $q_{i}$ son las variables observadas por el empleador pero no disponibles para el investigador, por ejemplo, información sobre entrevistas de trabajo y cartas de recomendación; $z_{i}$ son variables no observadas por los empleadores pero disponibles para el investigador, como puntajes de pruebas estandarizadas; $\eta_{i}$ son variables que no son observadas ni por el empleador ni por el investigador, o el término de error. $\mathrm{H}\left(x_{i}\right)$ resume el perfil de experiencia del logaritmo de productividad, que puede deberse a un proceso de inversión a lo largo del ciclo de vida.

Más adelante se explorarán las implicancias del modelo EL-SD en el mercado chileno. El primer efecto es que los coeficientes de regresión lineal de las habilidades no observadas $z$ aumentan con la experiencia. En otras palabras, con la experiencia se revela más información sobre las verdaderas habilidades de los trabajadores $\mathrm{y}$, por lo tanto, los salarios reflejan cada vez más la productividad, aumentando la correlación que existe entre salarios y habilidades. Por ende, se espera que la rentabilidad de las variables $z$ aumente con la experiencia. Un segundo efecto es que el rendimiento en función del prestigio de la universidad debería disminuir con la experiencia, dado que la experiencia reduce la importancia que los empleadores otorgan al prestigio de la universidad a la hora de predecir la productividad de sus trabajadores. La calidad y el prestigio de la universidad suben los ingresos al principio de la carrera porque las universidades de calidad aumentan la productividad, y también debido a la discriminación estadística. Sin embargo, al correr del tiempo disminuye la incidencia de la discriminación estadística sobre los salarios. Por lo tanto, permitir que la variable $\boldsymbol{z}$ interactúe con la experiencia reduce más los coeficientes de prestigio de la universidad para los trabajadores de más experiencia si se los compara a los trabajadores con menos experiencia. 
En este artículo se estima la siguiente ecuación logarítmica de ingresos. Ella permite interacciones lineales entre variables fáciles de observar y los puntajes estandarizados de la PSU con la experiencia:

$$
w_{i, t}=\beta_{0}+\sum_{j} \beta_{s_{j}} s_{j i, t}+\beta_{z} z_{i, t}+\sum_{j} \beta_{s_{j}, x}\left(s_{j i, t} \times x_{i, t}\right)+\beta_{z, x}\left(z_{i, t} \times x_{i, t}\right)+f\left(x_{i, t}\right)+\beta_{\Phi}^{\prime} \Phi_{i, t}+v_{i, t}
$$

donde $w_{i, t}$ es el logaritmo del salario de la persona i durante el período t, $s_{1, t}$ es una variable dummy para colegio secundario privado, $s_{2, t}$ hasta $s_{4, t}$ son variables dummies para rankings de universidades como proxy de prestigio y calidad de la universidad, $z_{i}$ es el puntaje obtenido en la PSU, $x_{i}$ son los años de experiencia en el mercado laboral, y $\Phi_{\mathrm{i}}$ es el vector de controles. Una importante característica de esta regresión es que muestra cómo la relación entre salarios y habilidades, salarios y colegios privados, y salarios y ranking de la universidad cambia con la experiencia.

Se utilizan las pruebas de lenguaje y matemática de la PSU como una forma de medir habilidades que no son fácilmente observadas por las empresas debido a diferentes razones. Primero, estas pruebas son los componentes de la PSU formulados para medir las habilidades inherentes de los postulantes y se administran de tal manera que posibles estudiantes de universidades muestran sus conocimientos y habilidades para tener éxito en la universidad. ${ }^{5}$ Segundo, los empleadores no tienen acceso al puntaje de la PSU al momento de fijar los salarios. No es común que los empleadores chilenos pidan el puntaje de la PSU en el proceso de la postulación (Swett, 2011). Esto podría explicarse porque no piensan que ésta entrega información importante más allá de la enseñanza media y de la universidad, o, contrariamente a lo que ocurre con las universidades, los empleadores no tienen acceso a la distribución completa de los puntajes de la PSU, por lo que el valor absoluto del puntaje en la PSU de un trabajador podría no ser muy informativo para una empresa.

A partir de la ecuación (2) podemos testear la discriminación estadística sobre la base de variables fáciles de observar si las interacciones entre la enseñanza media y la universidad con la experiencia son

5 Repetto \& Fischer (2003) muestran que las notas de la enseñanza media y las pruebas específicas de física y matemática de la PSU son variables predictivas de los resultados que se obtendrán en la escuela de ingeniería. 
no crecientes $^{6}$, y la interacción entre las habilidades y la experiencia son no decrecientes ${ }^{7}$. Obviamente, si los empleadores tienen toda la información sobre las habilidades y productividad de potenciales trabajadores, entonces $\beta_{s j, x}=\beta_{z, x}=0 .{ }^{8}$

\subsection{Datos}

Los datos usados en el estudio vienen de "Futuro Laboral", un proyecto del Ministerio de Educación chileno que hace un seguimiento de los egresados de programas de educación superior durante los primeros años de experiencia en el mercado laboral. Se tiene una base de datos de panel que vincula declaraciones de renta con las carreras y las instituciones de las que se graduaron. Sólo existen datos para quienes se graduaron; aquellos que abandonaron o que nunca se matricularon en la universidad no conforman la muestra. La información sobre ingresos está disponible para los años 1996 a 2005.

El Servicio de Impuestos Internos (SII) entrega datos sobre los ingresos anuales en base a las declaraciones de impuesto a la renta. Además de salarios, se sabe la ciudad o ciudades de empleo, número de empleadores y sector económico. Los datos demográficos, incluyendo edad, sexo, nombre de la institución donde se formaron los estudiantes, asignatura principal y el año de graduación, fueron proporcionados por el Ministerio de Educación (MINEDUC). Se usa una submuestra con datos adicionales recopilados por el MINEDUC con el puntaje de la PSU, las notas de la enseñanza media y el colegio de enseñanza media del que se graduaron los individuos.

El salario medido en la muestra es el ingreso anual obtenido por trabajos y servicios prestados por la persona en el sector formal. Se usa el índice de precios al consumidor (IPC) como deflactor para calcular

${ }^{6} \beta_{\mathrm{sj}, \mathrm{x}} \leq 0$.

${ }^{7} \beta_{\mathrm{z}, \mathrm{x}} \geq 0$.

${ }^{8}$ Las pruebas que AP desarrollaron podrían expresarse como:

$$
\frac{\partial \beta_{s_{j}, x}}{\partial x}=-\lambda_{z, s_{j}} \frac{\partial \beta_{s_{j}, x}}{\partial x}
$$

donde $\lambda z, s$ es el coeficiente de regresión de z sobre sj. Esta ecuación afirma que como sj es parte de la información inicial que los empleadores tienen acerca de la productividad de un nuevo trabajador, la disminución de $\beta$ sj,x sólo proviene de la relación entre sj y z. 
los salarios reales. La variable de habilidad, el puntaje de la PSU, se construye como un simple promedio del puntaje de las pruebas de lenguaje y matemática. A fin de facilitar la interpretación, se normalizó el puntaje de la PSU para que tuviera una media de cero y una desviación estándar de 1. La variable de la experiencia se calculó según el número de años en que una persona ha obtenido ingresos y pagado impuestos. Los sectores económicos se dividieron en doce categorías: agricultura, minería, sector manufacturero, construcción, negocios, servicios, bienes raíces, transporte, comunicaciones, servicios, educación y salud. Se excluyó a los administradores públicos dado que pertenecen al sector público con una estructura de salarios distinta. Los trabajadores independientes también fueron excluidos de la muestra.

Finalmente, para las variables de ranking de las universidades, se clasificaron todas las universidades utilizando el ranking de la revista Qué Pasa, que usa una metodología similar al ranking de universidades de U.S. News. Luego se agruparon las universidades en distintos niveles según su ranking y su tipo, es decir, dos niveles para las universidades tradicionales y dos niveles para las universidades privadas, donde el nivel 1 incluye a las universidades más antiguas y más prestigiosas de cada grupo. La tabla $n^{\circ} 1$ muestra el resumen estadístico de los datos. La tabla $\mathrm{n}^{\circ} 2$ entrega algunas estadísticas para clúster de ranking. Como era de esperar, los trabajadores egresados de universidades tradicionales del nivel 1 tienen puntajes en la PSU significativamente más

TABLA N 1: $\quad$ ESTADÍSTICA DESCRIPTIVA, "FUTURO LABORAL", 1995-2005

\begin{tabular}{lcccc}
\hline Variable & Media & Desviación estándar & Mínima & Máxima \\
\hline Salarios anuales reales & 5016960 & 4690400 & 128960 & 72369440 \\
Logaritmo de salarios & 15,16 & 1,0797 & 11,95 & 18,28 \\
anuales & 0,53 & 0,5 & 0 & 1 \\
Mujeres & 3,89 & 2,36 & 0 & 9 \\
Experiencia & 0,01 & 0,99 & $-4,33$ & 2,59 \\
$\begin{array}{l}\text { Puntaje estandarizado de } \\
\text { la PAA }\end{array}$ & 0,08 & 0,26 & 0 & 1 \\
\hline Colegio secundario privado & 0,59 & & \\
\hline
\end{tabular}

Los salarios reales están en pesos chilenos, año base 1995. 
TABLA $N^{\circ} 2$ : ESTADÍSTICA DESCRIPTIVA PARA EL RANKING DE UNIVERSIDADES

\begin{tabular}{lcccc}
\hline $\begin{array}{l}\text { Clúster de } \\
\text { universidades }\end{array}$ & Observaciones & Media PSU & $\begin{array}{c}\text { Desviación estándar } \\
\text { PSU }\end{array}$ & Colegio privado \\
\hline $\begin{array}{l}\text { Tradicionales } \\
\text { nivel 1 }\end{array}$ & 10.594 & 0,96 & & 0,12 \\
$\begin{array}{l}\text { Tradicionales } \\
\text { nivel 2 }\end{array}$ & 30.664 & $-0,087$ & 0,84 & 0,03 \\
Privadas nivel 1 & 5.180 & $-0,21$ & 0,94 & 0,23 \\
Privadas nivel 2 & 7.522 & $-1,02$ & 0,91 & 0,08 \\
\hline
\end{tabular}

altos que los trabajadores egresados de otras universidades ${ }^{9}$. Las instituciones privadas del nivel 1 tienen el mayor porcentaje de titulados provenientes de un colegio privado, $24 \%$.

\subsection{Discriminación estadística en base al colegio y al ranking de universidades}

Primero se estima la ecuación (2) sin incorporar la interacción del puntaje de la PSU con la experiencia ${ }^{10,11}$. Esta restricción implica que el efecto de las habilidades sobre el logaritmo del ingreso es constante durante el ciclo de vida. La tabla $\mathrm{n}^{\circ} 3$ muestra los resultados de la estimación usando distintas especificaciones. Las mujeres ganan considerablemente menos que los hombres, incluso cuando se controla por la carrera, región, sector económico, colegio, universidad y puntaje de la PSU. En efecto, las mujeres muestran salarios en promedio $22,6 \%$ inferiores al de los hombres.

La columna 1 de la tabla $\mathrm{n}^{\circ} 3$ entrega los resultados de la estimación cuando se excluye la interacción de las habilidades (puntaje en la PSU) con la experiencia. Vemos que un aumento de una desviación

${ }^{9}$ Dado que uniformicé los puntajes de la PSU para que tuvieran una media de 0 y una desviación estándar de 1 , es posible tener puntajes de la PSU inferiores a 1, es decir, puntajes bajo la media.

${ }^{10} \beta_{z j, x}=0$.

${ }^{11}$ Durante todo el análisis, las variables del colegio secundario privado (s1), las variables ficticias de ranking de universidades $\left(s_{2}-s_{4}\right)$ y las pruebas de PSU estandarizadas promedio $(z)$ se hacen interactuar con la experiencia dividida por 10. Por tanto, los coeficientes de estos términos de interacción capturan el cambio en la curva de salarios entre $\mathrm{x}=0 \mathrm{y} \mathrm{x}=10$. 
TABLA N ${ }^{\circ}$ 3: $\quad$ LOS EFECTOS DE UNA PSU ESTANDARIZADA, RANKING DE COLEGIOS SECUNDARIOS Y UNIVERSIDADES SOBRE LOS SALARIOS

\begin{tabular}{|c|c|c|c|c|}
\hline Variables & (1) & (2) & (3) & (4) \\
\hline \multirow[t]{2}{*}{ Mujeres } & $-0,256^{* * *}$ & $-0,256 * * *$ & $-0,256^{* * *}$ & $-0,256^{* * *}$ \\
\hline & $(0,0187)$ & $(0,0187)$ & $(0,0187)$ & $(0,0186)$ \\
\hline Puntaje estandarizado de la & $0,0938^{* * *}$ & $0,0597 * * *$ & $0,0939 * * *$ & $0,0436 * *$ \\
\hline PSU & $(0,0128)$ & $(0,0173)$ & $(0,0128)$ & $(0,0205)$ \\
\hline \multirow[t]{2}{*}{ Colegio secundario privado } & $0,158 * * *$ & $0,170 * * *$ & $0,168 * * *$ & $0,183 * * *$ \\
\hline & $(0,0296)$ & $(0,0300)$ & $(0,0303)$ & $(0,0308)$ \\
\hline Universidades tradicionales & $0,160 * * *$ & $0,161 * * *$ & $0,125 * * *$ & $0,214 * * *$ \\
\hline nivel 1 & $(0,0368)$ & $(0,0369)$ & $(0,0474)$ & $(0,0571)$ \\
\hline Universidades tradicionales & 0,0449 & 0,0458 & 0,0185 & 0,0697 \\
\hline nivel 2 & $(0,0289)$ & $(0,0289)$ & $(0,0418)$ & $(0,0462)$ \\
\hline Universidades privadas & $0,0798 * * *$ & $0,0806^{* * *}$ & 0,00731 & 0,0400 \\
\hline nivel 1 & $(0,0297)$ & $(0,0298)$ & $(0,0448)$ & $(0,0455)$ \\
\hline Colegio secundario privado $\mathrm{x}$ & $-0,024$ & $-0,0565$ & $-0,0499$ & $-0,0897$ \\
\hline experiencia & $(0,0503)$ & $(0,0514)$ & $(0,0530)$ & $(0,0557)$ \\
\hline Universidades tradicionales de & & & 0,0848 & $-0,1310$ \\
\hline nivel $1 \mathrm{x}$ experiencia & & & $(0,0764)$ & $(0,1030)$ \\
\hline Universidades tradicionales de & & & 0,0637 & $-0,0571$ \\
\hline nivel $2 \mathrm{x}$ experiencia & & & $(0,0700)$ & $(0,0814)$ \\
\hline Universidades privadas de & & & $0,175^{* *}$ & 0,0973 \\
\hline nivel $1 \mathrm{x}$ experiencia & & & $(0,0868)$ & $(0,0873)$ \\
\hline Puntaje estandarizado de la & & $0,0838 * * *$ & & $0,123 * * *$ \\
\hline PSU $x$ experiencia & & $(0,0255)$ & & $(0,0359)$ \\
\hline \multirow[t]{2}{*}{ Constante } & $12,21 * * *$ & $12,22 * * *$ & $12,24 * * *$ & $12,20 * * *$ \\
\hline & $(0,1990)$ & $(0,1990)$ & $(0,2010)$ & $(0,2030)$ \\
\hline Experiencia cúbica & Sí & Sí & Sí & Sí \\
\hline Observaciones & 38.482 & 38.482 & 38.482 & 38.482 \\
\hline $\mathrm{R}$ al cuadrado & 0,353 & 0,354 & 0,353 & 0,354 \\
\hline
\end{tabular}

La experiencia se modela con un polinomio cúbico. Las especificaciones también incluyen controles para carreras, regiones geográficas, sectores económicos y efectos del tiempo.

Los errores estándar (entre paréntesis) son White/Huber para controlar una posible heterocedasticidad y correlación entre varias observaciones por individuo.

$* * *$ p $0,01, * *$ p $0,05, *$ p 0,1 
estándar en el puntaje estandarizado de las pruebas de la PSU implica un aumento en promedio de $9,8 \%$ en el salario, ceteris paribus. Asistir a un colegio secundario privado tiene un efecto positivo e importante sobre los ingresos para titulados de la universidad, aumentando el salario en $17 \%$. Este resultado es interesante porque sólo estamos examinando a los titulados de la universidad, pero no es sorprendente para el mercado laboral chileno dada la práctica común de incluir información sobre el colegio en el curriculum vitae. En principio, esto podría permitir a todos los empleadores discriminar a los trabajadores en base al colegio. Aquí la variable dummy del colegio privado puede captar otros efectos, tales como el nivel socioeconómico de los padres, lo que puede ser un factor importante en la calidad de las redes sociales de una persona para encontrar mejores trabajos. La interacción del coeficiente del colegio privado y la experiencia que nos permite ver el efecto de asistir a un colegio de enseñanza media privado al correr del tiempo es $-0,024$, lo que significa que el efecto de haber asistido a un colegio privado sobre el salario disminuye con la experiencia.

En la columna 2 se incluye la interacción entre el puntaje de la PSU y la experiencia. El efecto de una variación de una desviación estándar en los puntajes de la PSU aumenta los salarios 6,15\% justo después de graduarse. Haber asistido a un colegio secundario privado aumenta los salarios logarítmicos en 0,17 , es decir, cuando se incorpora la habilidad en el tiempo (puntaje de la PSU X experiencia), aquellos que asistieron a un colegio secundario privado tienen un salario en promedio 18,5\% más alto. El coeficiente de colegios secundarios privados interactuado con la experiencia en esta segunda especificación cae fuertemente a $-0,0565$. Por ende, el efecto implícito de ir a un colegio secundario privado en vez de a un colegio secundario público disminuye de 0,170 a 0,1135 en diez años. Estos resultados respaldan la hipótesis de la discriminación estadística dado que los empleadores también basan sus suposiciones previas sobre las habilidades en información del colegio secundario al que asistieron los trabajadores ${ }^{12}$. Es importante recordar que estos resultados se aplican a los titulados de la universidad; por tanto, encontramos que incluso después de ir a la universidad, el colegio secundario al que asistió una persona es un determinante sig-

${ }^{12}$ Los colegios secundarios privados son mucho más caros y selectivos que las escuelas públicas en Chile. 
nificativo de sus remuneraciones, especialmente al iniciarse la carrera de un trabajador ${ }^{13}$.

En la columna 3 de la tabla $n^{\circ} 3$ se introduce la interacción entre las variables dummies de la universidad y la experiencia, excluyendo el efecto de la interacción entre las habilidades (puntaje de la PSU) y la experiencia. Los rankings de universidades entregan una señal sobre la calidad de la universidad a los empleadores, lo que, a su vez, se utiliza para hacer suposiciones sobre la productividad de los trabajadores. Por lo tanto, se cree que personas graduadas de instituciones prestigiosas sean más productivas, por lo que sus ingresos deberían ser más altos por lo menos al inicio de sus carreras respecto de titulados de instituciones menos prestigiosas. A medida que pasa el tiempo, los empleadores deberían basarse cada vez menos a la hora de inferir la productividad en la universidad de la que egresó el trabajador puesto que pueden observar su desempeño. Los resultados de la regresión sugieren que para las universidades pertenecientes al grupo de las tradicionales más antiguas, el efecto sobre los ingresos es positivo, importante y estadísticamente significativo $^{14}$.

El resultado más importante de la tabla $\mathrm{n}^{\circ} 3$ viene de la columna 4, donde se incluye la interacción entre las variables dummies del ranking de universidades y la experiencia, y la interacción entre el puntaje de la PSU y la experiencia. Resulta que el efecto de una variación de una desviación estándar en el puntaje de la PSU aumenta los salarios $4,5 \%$ justo después de la graduación. Cabe destacar que el efecto de la interacción de la PSU y la experiencia es importante y estadísticamente significativo. De hecho, una variación de una desviación estándar en los puntajes de la PSU aumenta los salarios 13\% después de diez años en el mercado laboral. Estos resultados captan la idea de que los empleadores van aprendiendo a medida que observan las habilidades de sus trabajadores, lo que se ve reflejado en los salarios.

Las estimaciones de los coeficientes de colegio secundario privado y su interacción con la experiencia aumentan respecto de las estimaciones de la columna 2. Es decir, el efecto de asistir a un colegio secundario privado incrementa los salarios en aproximadamente $20 \%$

${ }^{13}$ Ver Núñez y Gutiérrez (2004) que presentan resultados similares, aun cuando utilizan datos sólo de una universidad. Véase también los resultados obtenidos por Contreras, Rodríguez y Urzúa (2013).

${ }^{14}$ La variable ficticia omitida es Nuevas Universidades Privadas. 
para los trabajadores sin experiencia y sólo aumenta 9,8\% los salarios para aquellos con experiencia (diez años después).

Respecto del efecto del prestigio de la universidad sobre los sueldos, la columna 4 muestra que sólo el nivel 1 de las universidades tradicionales importa en materia de salarios, dado que es el único coeficiente importante y estadísticamente significativo. Es decir, al principio de la carrera de un trabajador se puede esperar un sueldo aproximadamente $24 \%$ superior cuando el trabajador viene de una universidad tradicional de nivel 1 comparado con una universidad privada nivel 2, pero diez años después el efecto disminuye a $8,7 \%$. Por ende, el efecto sobre los ingresos de ir a una universidad de nivel 1 disminuye significativamente con el tiempo, apoyando la teoría del modelo SD-EL ${ }^{15}$. Los coeficientes de las universidades tradicionales de nivel 2 y de las universidades privadas de nivel 1 son más pequeños y estadísticamente no significativos. Por lo tanto, no existe evidencia de discriminación estadística ni de aprendizaje del empleador para las universidades tradicionales de nivel 2 o las universidades privadas de nivel 1 comparadas con las universidades privadas de nivel 2 .

Finalmente, el coeficiente de 0,0436 del puntaje de la PSU y el coeficiente de 0,123 del puntaje de la PSU interactuado con la experiencia sugieren que una variación de una desviación estándar del puntaje estandarizado de la PSU aumenta su efecto de $4,5 \%$ a $17 \%$ en diez años, consistentemente con la tesis de aprendizaje del empleador que indica que los salarios reflejan cada vez más la productividad, aumentando la correlación entre salarios y habilidades. Se podría sostener que el coeficiente positivo sobre la interacción del puntaje de la PSU y la experiencia podría atribuirse a una asociación entre puntaje de la PSU y capacitación en el trabajo. No obstante, resulta difícil conciliar este argumento con el hecho de que el coeficiente de la interacción de las universidades tradicionales y la experiencia es negativo. En conclusión, los

${ }^{15}$ El hecho de que el coeficiente de las universidades multiplicado por la experiencia es negativo mientras el coeficiente de la PSU estandarizada multiplicado por la experiencia es positivo disminuye la posibilidad de que se asocie este último con el entrenamiento. Existen pruebas empíricas de modelos de capital humano y entrenamiento en el trabajo que sugieren que es muy probable que los trabajadores más educados y capaces reciban más entrenamiento. Si éste es el caso, entonces esperamos que el efecto sobre los salarios tanto del puntaje de la PSU como de ir a la universidad aumente con el tiempo. 
resultados de las regresiones indican que en el mercado laboral chileno existe evidencia de una discriminación estadística en base al colegio y la universidad, sobre todo si el trabajador egresó de una universidad tradicional de nivel 1 en Chile.

\section{El modelo de aprendizaje del empleador}

En esta sección se estudian los perfiles de salarios suponiendo que las diferencias de salarios observadas se deben totalmente a la discriminación estadística basada en el ranking de la universidad. Los resultados de la sección 3 sugieren que obtener un título de una universidad tradicional de nivel 1 incrementa los salarios considerablemente respecto de una universidad privada de nivel 2. Es decir, egresar de las mejores universidades les permite a los trabajadores revelar sus habilidades a los empleadores al momento de ingresar al mercado laboral. Por ello se construye y estima un modelo de aprendizaje del empleador que incorpora esta percepción.

El modelo se basa en Altonji y Pierret (2001), Lange (2007) y Arcidiacono et al. $(2010)^{16}$. Los salarios pueden describirse mediante la siguiente ecuación por cada año de experiencia $x$ :

$$
w_{x}=\lambda_{x}\left\{\left(1-\Theta_{x}\right) \overline{P S U}+\Theta_{x} P S U\right\}+k_{x}
$$

El modelo arroja una ecuación que relaciona los salarios logarítmicos y una función lineal de la habilidad del trabajador (puntaje de la PSU, que inicialmente no se observa), y el puntaje promedio de la PSU del grupo al que pertenece. Los empleadores usan las habilidades de los trabajadores para fijar los salarios, pero dado que estas habilidades se revelan gradualmente, los empleadores dan considerablemente más peso a la productividad promedio de los egresados de distintos niveles de universidades. Al principio de la carrera laboral no se puede observar la productividad individual, pero con el tiempo los empleadores van incorporando más información sobre sus trabajadores y dan menos importancia a las habilidades promedio de los trabajadores procedentes de ciertas universidades y más importancia a las habilidades individuales a medida que éstas se pueden observar. modelo.

${ }^{16}$ Véase la sección Anexo, donde se presenta la derivación formal del 
La ecuación (3) implica que la importancia que los empleadores dan a las habilidades de un trabajador (puntaje obtenido en la PSU) en el tiempo x es $\Theta_{x}$ y la importancia del puntaje promedio de la PSU para egresados de un cierto grupo o nivel de universidades es $\left(1-\Theta_{x}\right)$. Para simplificar la comprensión de la dinámica del aprendizaje del empleador, se divide la muestra en dos grupos: los egresados de una universidad tradicional de nivel 1 y los egresados de todas las otras universidades. El objetivo consiste en determinar el parámetro de aprendizaje $\Theta_{x}$. Nótese que la importancia que los empleadores dan a las habilidades individuales puede aumentar con el tiempo a medida que los empleadores observan las habilidades individuales, captadas por $\Theta_{x}$ y porque la propia productividad de las habilidades aumenta con el tiempo, captada por $\lambda_{x}$ (verdadera productividad). En el momento $\mathrm{x}=0$, si los empleados no observan nada relacionado con el puntaje individual de la PSU, entonces se basan en los promedios del grupo para fijar los salarios y $\Theta_{x}=0$. Con el tiempo, los empleadores observan más señales acerca de la productividad del trabajador y $\Theta_{x}$ aumentará en tanto que $\left(1-\Theta_{x}\right)$ disminuirá. La otra parte de la importancia dada al puntaje promedio de la PSU y al puntaje individual de la PSU viene del verdadero valor de la productividad de las habilidades, $\lambda_{x}$. Supongamos que la habilidad es menos importante para la productividad en trabajos iniciales que para trabajos adquiridos más adelante en la trayectoria de la carrera laboral. Entonces, $\lambda_{x}$ tendrá un menor valor inicialmente y aumentará con el tiempo, lo que da a entender que los empleadores dan más peso tanto al promedio de la PSU como a la PSU individual ${ }^{17}$.

Después de un desarrollo algebraico ${ }^{18}$, se puede escribir la ecuación (3) como:

$$
w_{i, x}=\beta_{x, S} \mathrm{~s}+\beta_{x, \text { Univ }} \text { Univ }+\beta_{x, P S U} P S U+\beta_{\Phi}^{\prime} \Phi_{i, t}+\beta_{x,}^{\prime}+\varepsilon_{x}
$$

La estimación de esta ecuación es directa dado que incluye una variable dummy Univ que toma el valor de 1 si el trabajador no egresó de una universidad tradicional de nivel 1. Los empleadores ponderan el ranking de la universidad principalmente desde dos fuentes: el término

${ }^{17}$ Nótese que si $\lambda_{\mathrm{x}}$ aumenta lo suficientemente rápido, la ponderación de la PSU aumentaría con el tiempo aun cuando el aprendizaje tendería a reducirla, lo que ocurre si $\lambda_{x}\left(1-\Theta_{x}\right)>\lambda_{x}-1\left(1-\Theta_{x-1}\right)$.

${ }^{18}$ Véase en Arcidiacono et al. (2010) un desarrollo de álgebra similar. 
$\left(1-\Theta_{x}\right)$ que capta el aprendizaje de las habilidades, y el término $\lambda_{x}$ que capta la variación del valor de la verdadera productividad de las habilidades $^{19}$.

Una vez estimada la ecuación (4), se puede estimar $\lambda_{x}$ y $\Theta_{x}$ :

$$
\begin{aligned}
& \lambda_{x}=\beta_{x, P S U}-\beta_{x, \text { Univ }} /\left(\overline{P S U}_{\text {Univ }}-\overline{P S U_{\text {Top-Univ }}}\right) \\
& \Theta_{x}=\frac{\beta_{x, P S U}}{\beta_{x, P S U}-\beta_{x, \text { Univ }} /\left(\overline{P S U_{\text {Univ }}}-{\overline{P S U_{\text {Top-Univ }}}}\right)}
\end{aligned}
$$

Los resultados de la estimación se exhiben en los gráficos $n^{\circ} 3$. Los dos primeros gráficos muestran los coeficientes estimados de Univ (titulados de universidades tradicionales que no son de nivel 1) y puntaje estandarizado de la PSU para cada nivel de experiencia mostrando el 95 por ciento de intervalo de confianza en gris. La diferencia del salario inicial debida al ranking de la universidad es de 16 por ciento, que desaparece casi completamente en nueve años. El efecto de un aumento de una desviación estándar en el puntaje de la PSU empieza inicialmente en 5 por ciento, y llega hasta 13,5 por ciento después de seis años y luego disminuye.

Los dos últimos gráficos presentan el parámetro de aprendizaje $\Theta_{x}$, y el parámetro de productividad real $\lambda_{x}$. Se usan los perfiles de experiencia para calcular cuánto del cambio en los salarios se debe al ranking de la universidad, a los puntajes estandarizados de la PSU y la productividad real de las habilidades y cuánto puede atribuirse al aprendizaje del empleador. Uno muestra que el parámetro $\Theta_{x}$ llega a 0,5 en tres años, lo que significa que en tres años los empleadores observan alrededor del 50 por ciento de las habilidades de los trabajadores captadas por el puntaje estandarizado de la PSU. Respecto de la velocidad del aprendizaje, se podría decir que los empleadores chilenos aprenden rápidamente ${ }^{20}$. Por otra parte, la productividad real de las habilidades de un trabajador, captadas por el puntaje estandarizado de

${ }^{19}$ Nótese que el tamaño y el valor del coeficiente de Univ depende totalmente del perfil de experiencia de $\lambda_{\mathrm{x}} \mathrm{y} \Theta_{\mathrm{x}}$.

${ }^{20}$ Lange (2007) muestra que en EE. UU. los empleadores reducen el error de expectativas inicial en un $50 \%$ en los tres primeros años. 
GRÁFICOS N ${ }^{\circ}$ 3: EVOLUCIÓN DEL EFECTO DEL RANKING DE LAS UNIVERSIDADES Y DEL PUNTAJE DE LA PSU SOBRE LOS SALARIOS, EL PARÁMETRO DE APRENDIZAJE $\Theta_{\mathrm{X}}$, Y LA PRODUCTIVIDAD REAL DE LA PSU $\lambda_{\mathrm{X}}$

Coeficiente de Univ (u. tradicionales nivel 2)
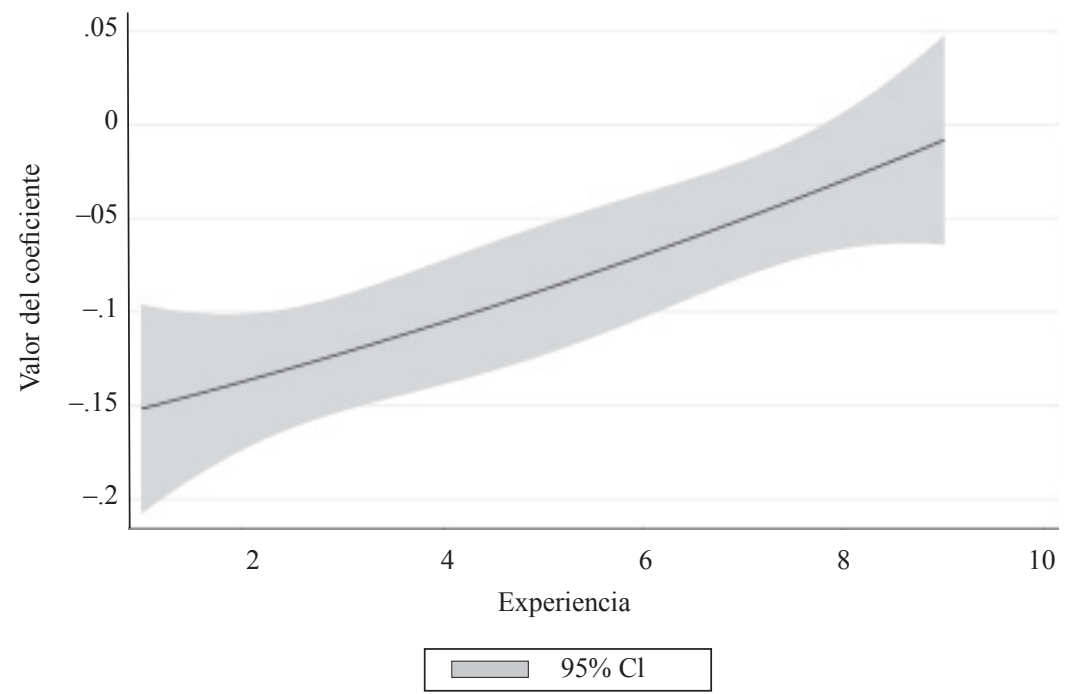

Coeficiente de PSU

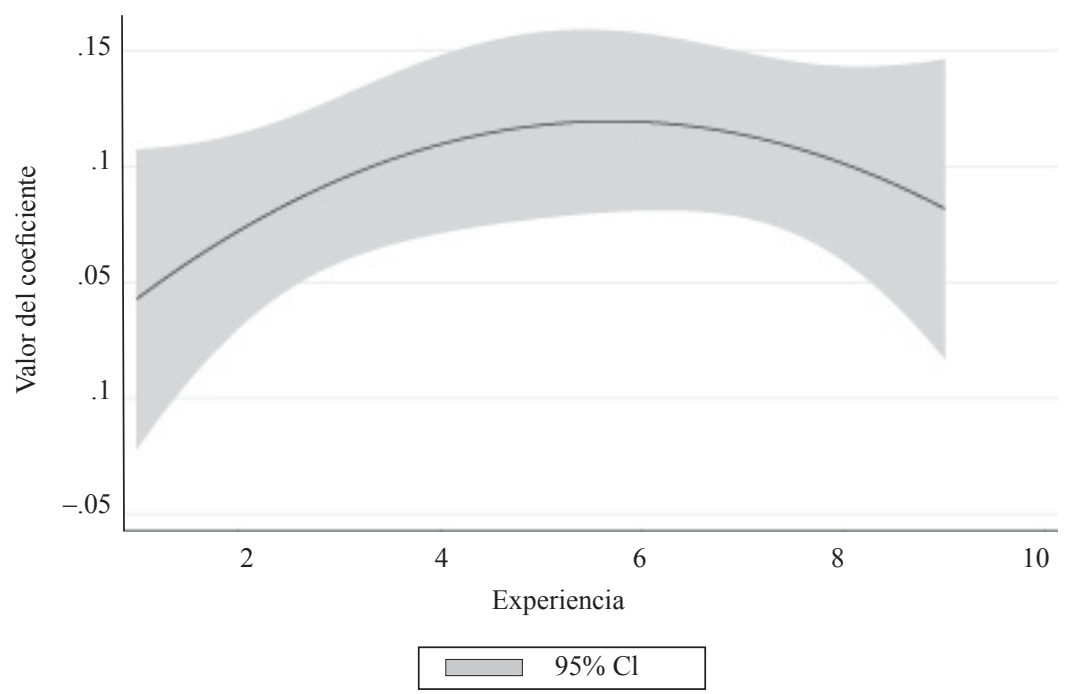


Parámetro de aprendizaje theta
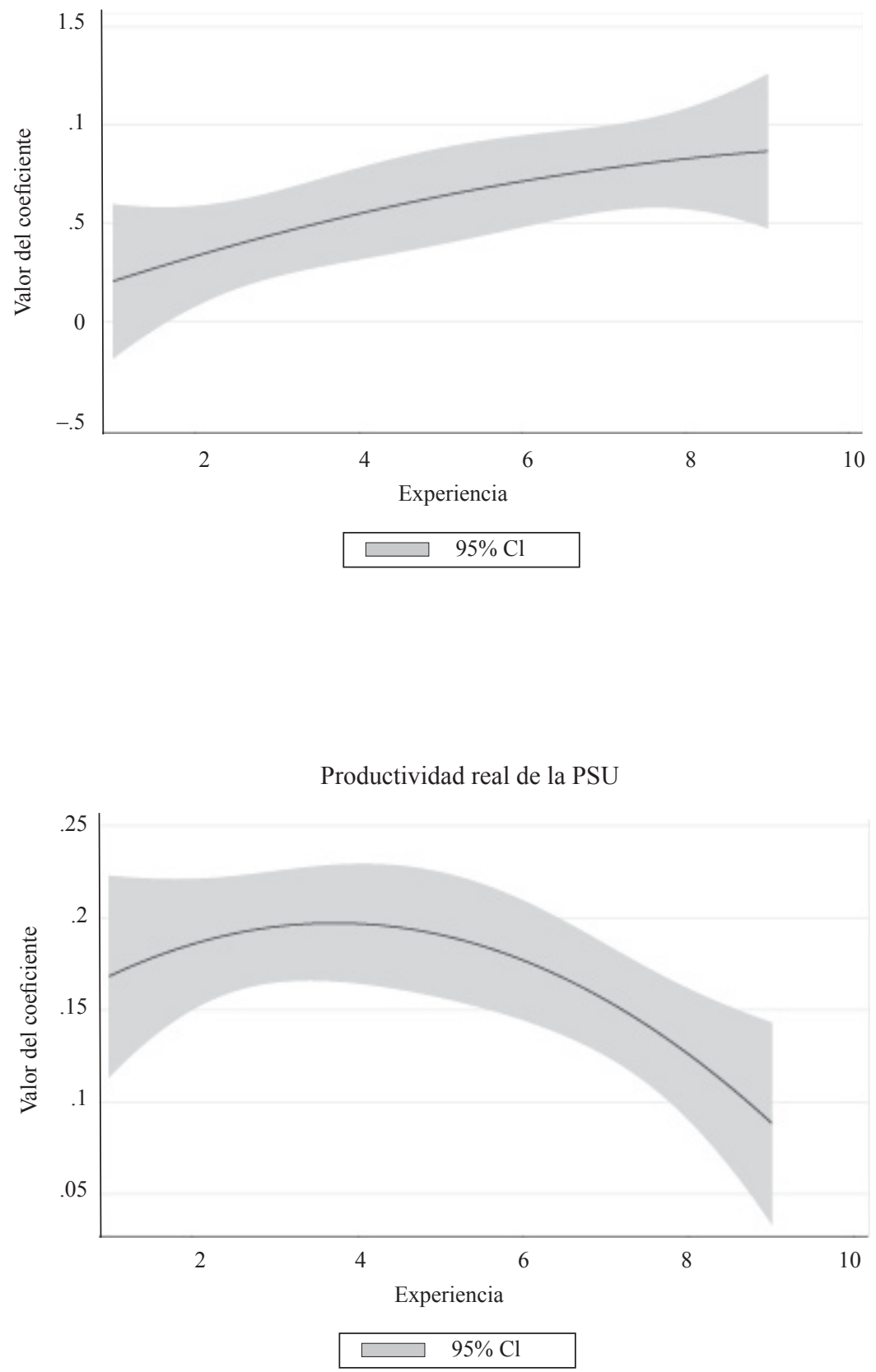
la PSU, inicialmente aumenta con la experiencia, pero luego disminuye (después de cinco años).

La ponderación que los empleadores dan a Univ en la regresión del salario debido al aprendizaje del empleador es $\left(1-\Theta_{x}\right)$, que comienza en 1,0 y disminuye a 0,5 después de tres años. Dado que los empleadores inicialmente no pueden observar las habilidades directamente, confían en el puntaje promedio de la PSU de la universidad de donde egresó el trabajador para estimar su productividad. Con el tiempo, los empleadores pueden observar la productividad del trabajador y tienen menos incentivos para discriminar estadísticamente, dándole menos peso al puntaje promedio de la PSU y más peso a las habilidades observadas.

\section{Consecuencias en materia de políticas públicas}

Mis resultados confirman que en el contexto del mercado laboral chileno se enfatiza demasiado el colegio y el ranking de las universidades al fijar salarios. Ambos tienen un efecto desmedido sobre los salarios, incluso después de controlar las habilidades, carrera, género, región geográfica y sector económico. Estos hallazgos se pueden explicar por la falta de información o información incompleta: los empleadores no pueden observar la productividad potencial del empleado y usan los colegios y los rankings de la universidad como señales de productividad. Por lo tanto, sería útil que potenciales empleadores tuviesen más información pertinente para evaluar la productividad de un potencial empleado.

Los resultados de este artículo respaldan la conclusión de que el puntaje de la PSU puede entregar información significativa sobre la productividad de un potencial empleado, que no se capta a través de los rankings del colegio o de la universidad u otras variables de control. Por ende, incorporar el puntaje de la PSU en los curriculum vitae de los trabajadores podría reducir la brecha informativa entre trabajadores y empleadores, disminuyendo la discriminación estadística basada en colegios secundarios y rankings de la universidad. No obstante, dado que el puntaje de la PSU no es informativo sin una adecuada información sobre la distribución del puntaje, potenciales empleados y empleadores se verían beneficiados si en los curriculum vitae se incluyera rankings basados en el percentil de puntajes de la PSU. 
La discriminación estadística en Chile basada en el ranking de colegios secundarios y de las universidades no desaparecerá completamente al incorporarse rankings basados en el percentil de puntajes de la PSU. Existen otras características que pueden señalarse por el colegio y universidad que pueden valorarse en el mercado laboral, tales como la ambición, la afiliación religiosa o clase social, que no pueden medirse en la PSU.

Considerando que el ranking basado en el percentil de puntajes de la PSU no es una medición perfecta, podrían también usarse otras mediciones útiles para medir las habilidades y disminuir así el efecto de la discriminación estadística en Chile. Estas otras mediciones podrían proporcionar una mejor proxy para habilidades específicas valoradas en el mercado laboral, tales como la ambición, habilidades manuales u otras no cognitivas.

\section{Conclusiones}

Este estudio analiza los efectos sobre los ingresos del ranking de la universidad y de haber asistido a un colegio secundario privado en el mercado laboral de los egresados universitarios en Chile. También examina los efectos de la discriminación estadística y el aprendizaje del empleador sobre los salarios. Los empleadores usan el ranking de la universidad y el de los colegios para hacer suposiciones sobre la productividad de los trabajadores. Con el paso del tiempo, los empleadores reúnen información sobre la productividad de sus trabajadores y reajustan sus estimaciones iniciales, un proceso conocido como aprendizaje del empleador. A medida que avanza el proceso de aprendizaje, el modelo predice que los salarios deberían determinarse menos en función del ranking de la universidad y del colegio y más en función de las habilidades.

Según los modelos tradicionales de señalización de las habilidades, la educación les permite a las personas clasificarse en grupos correlacionados con la habilidad. Por consiguiente, indicar la universidad de la cual egresó la persona debería constituir una fuerte señal de productividad, sin embargo vemos en los datos que las habilidades se van revelando gradualmente. Por ende, es económicamente relevante saber cuánto demora que los egresados de la universidad revelen sus verdaderas habilidades. A partir de la estimación del modelo de aprendizaje del empleador se puede concluir que los empleadores al fijar los salarios 
reducen en un 50 por ciento el peso que le dan al ranking de la universidad al cabo de tres años. Estos resultados sugieren que incorporar el percentil del puntaje de la PSU (prueba estandarizada de ingreso a la universidad en Chile) en el curriculum vitae de los trabajadores podría reducir la brecha informativa entre trabajadores y empleadores, disminuyendo la discriminación estadística basada colegios secundarios y en el ranking de la universidad. Esta medida podría mejorar la capacidad del mercado laboral chileno para asignar apropiadamente los salarios.

\section{Anexo \\ Derivación formal del modelo de aprendizaje del empleador}

El logaritmo de la productividad en el mercado laboral $\chi_{\mathrm{ix}}$ de un individuo i con $x$ años de experiencia podría especificarse como:

$$
\chi_{i x}=\alpha s_{i}+\beta q_{i}+\gamma z_{i}+\eta_{i}+H\left(x_{i}\right)
$$

donde $s_{i}, q_{i}, z_{i}$ y $\eta_{i}$ se definen como en la ecuación (1) de la sección 3. El efecto de $(q, z, \eta)$ sobre el logaritmo de productividad es captado por el parámetro $\lambda_{x}$. $H\left(x_{i}\right)$ resume el perfil de experiencia del logaritmo de productividad, lo que puede deberse a un proceso de inversión a lo largo del ciclo de vida.

El modelo necesita tres supuestos importantes. Primero, los empleadores predicen la productividad en base a las variables $s_{i}, q_{i} \mathrm{y}$ las señales que obtienen con el tiempo. Segundo, la parte no observada de las habilidades y la información que los empleadores tienen inicialmente no pueden usarse para predecir $z_{i}$. Tercero, todos los empleadores poseen la misma información, es decir, información simétrica ${ }^{21}$.

La idea del modelo es interpretar la variación del gradiente de experiencia atribuible al proceso de aprendizaje del empleador. Por consiguiente, podemos concentrarnos en el problema que enfrentan las empresas que tratan de predecir la productividad de los trabajadores en

${ }^{21}$ Por otra parte, el supuesto de la información simétrica podría validarse mediante el trabajo de Schönberg (2007). Éste encuentra que el aprendizaje del empleador es casi simétrico en los EE. UU. Por supuesto, esto podría no ser el caso de Chile. 
base a $s$ y $q$ y otra información que se va revelando a medida que los trabajadores pasan más tiempo en el mercado laboral. En otras palabras, las empresas resuelven un problema de extracción de señales. Este problema va variando en el tiempo a medida que se dispone de mayor información. Debido a restricciones de datos, este artículo no considera otras variables que no sean las habilidades innatas que afectan la productividad en el tiempo, como la capacitación en el trabajo.

Los empleadores no observan $z$, pero suponemos que observan la productividad promedio del grupo de trabajadores al que pertenece el trabajador $\bar{z}=E(\mathrm{z} \mid s, x$, Univ, ranking $)$. En este caso se hace el supuesto de que los empleadores conocen el puntaje promedio de la PSU para cada ranking de universidades ${ }^{22}$. Por lo tanto, los empleadores pueden predecir $z=\bar{z}+\varepsilon$.

Las empresas no observan $\chi$, pero sí observan una señal ruidosa de la productividad de los trabajadores, $y_{x}=z+\eta+\varepsilon_{x}$. Las distintas señales adicionales disponibles provienen de la experiencia en el mercado laboral, $Y^{x}=\left(y_{0}, y_{1} \ldots, y_{x-1}\right)$. En cada período $x$ el empleador observa $y_{x}=z+\eta+\varepsilon_{x}$, de manera que el número de mediciones adicionales viene de la experiencia de las personas.

Durante el primer período, $x=0$, la media de lo que suponen los empleadores acerca de $z+\eta$ es $\mu_{0}=\bar{z}+\alpha_{1} s+\alpha_{2} q$. Después de $x$ número de años, la distribución posterior $\mu_{\mathrm{x}}=\left(1-\theta_{x}\right) \mu_{x-1}+\theta_{x} y_{x}$ donde $\theta_{x}$ es la ponderación bayesiana óptima que los empleadores usan para el cálculo de la media anterior. Al tiempo $x$, las expectativas de los empleadores sobre la productividad de los trabajadores es:

$$
E_{x}\left[\chi \mid \bar{z}, s, q, \mathrm{Y}^{x}\right]=r s+\lambda_{x} q+\lambda_{x}\left[\left(1-\theta_{x}\right) \mu_{x-1}+\theta_{x} y_{x}\right]+\mathrm{H}(\mathrm{x}) .
$$

Con el tiempo, los empleadores aprenden sobre la productividad real de los trabajadores, el término $\left[\left(1-\theta_{x}\right) \mu_{x-1}+\theta_{x} y_{x}\right]$ converge a $(\mathrm{q}+z+\eta)$ y el error de expectativas cae a cero.

Suponiendo competencia perfecta, información simétrica, empresas neutrales al riesgo y un mercado spot de mano de obra, se tiene que los salarios corresponden a la esperanza de la productividad condicional al momento $x$ :

${ }^{22}$ En Chile, los puntajes de corte de la PSU son públicos para cada carrera de cada universidad. Por lo tanto, los empleadores deberían tener por lo menos información del puntaje de la PSU mínimo que pide cada universidad. 
$W_{x}\left(\bar{z}, s, q, \mathrm{Y}^{x}\right)=E_{x}\left[\exp (\chi) \mid \bar{z}, s, q, \mathrm{Y}^{x}\right]=\exp \left(\mathrm{E}\left[\chi \mid \bar{z}, s, q, y^{x}\right]+\frac{1}{2} \sigma_{\mathrm{x}}^{2}\right)$

dado que la distribución de $\left(\chi \mid s, q, Y_{x}\right)$ es normal ${ }^{23}$. Tomando logaritmos, obtenemos el proceso de salarios logarítmicos:

$$
\begin{aligned}
& w_{x}\left(\bar{z}, s, q, \mathrm{Y}^{x}\right)=\lambda_{x}\left[\left(1-\theta_{x}\right) \mu_{x-1}+\theta_{x} y_{x}\right]+\mathrm{C}_{\mathrm{x}} \\
& \mathrm{C}_{x}=r s+\lambda_{x} q+H(x)+\frac{1}{2} \sigma_{\mathrm{x}}^{2}
\end{aligned}
$$

La ecuación (iv) vincula el logaritmo del salario con la información $\left(\bar{z}, s, q, Y^{x}\right)$ de que disponen las empresas. Esta ecuación también mide las señales del logaritmo de la productividad $Y^{x}$, que serán accesibles a lo largo del ciclo de vida del trabajador. Nótese, sin embargo, que en los datos se tiene $(\bar{z}, z, s, x)$ en vez de $\left(\bar{z}, s, q, Y^{x}\right)$, por ende, se define $\mathrm{q}=\gamma_{1} \mathrm{~s}+v_{1} \mathrm{y} \eta=\gamma_{2} \mathrm{~s}+v_{2}$. Adicionalmente, se define una proyección lineal de los salarios logarítmicos en $s$ y $z$ para distintos niveles de experiencia x. Usando la misma anotación que Lange (2007), tenemos que la proyección lineal de salarios logarítmicos condicionada por $(\bar{z}, z, s, x) \mathrm{es}^{24}$.

$$
E^{*}\left[w_{x} \mid s, z\right]=\lambda_{x}\left[\left(1-\theta_{x}\right) E^{*}\left[\mu_{x-1} \mid z, s\right]+\theta_{x} E^{*}\left(y_{x} \mid s, z\right)\right]+c_{x}
$$

Sustituyendo $\mu_{x}$ y q, se obtiene la siguiente expresión para el logartimo de los salarios para $x=1$

$$
\left.w_{1}=\lambda_{x}\left[\left(1-\theta_{1}\right) \bar{z}+\theta_{1} z\right)\right]+k_{1}
$$

donde $k_{l}=\lambda_{l}\left(1-\theta_{1}\right)\left[\alpha_{l} s+\alpha_{2}\left(\gamma_{1} s+v_{1}\right)\right]+c_{1}$.

Por lo tanto, los salarios logarítmicos en un período $x=1$ podrían verse como un promedio ponderado del puntaje de la PSU medio del grupo y de la PSU individual más una constante. Nótese que los componentes de $k_{l}$ indican que las expectativas de los empleadores acerca

${ }^{23}$ Recuerde que si $\mathrm{X}$ está normalmente distribuido con $\mu$ media y una varianza $\sigma^{2}$, entonces $E\left[e^{t X}\right]=\mathrm{e}^{\mu t \frac{1}{2} \sigma^{2} \tau^{2}}$. El error de expectativas en x es independiente de $(\bar{z}, s, z, q, \eta)$ y $\mathrm{Y}^{x}$, lo que implica que $\frac{1}{2} \sigma_{x}^{2}$ es constante.

${ }^{24}$ La proyección lineal de $\mathrm{x}$ en y se escribe como $E^{*}(x \mid y)$. 
de las habilidades de los trabajadores dependen de las habilidades promedio $z$, de las variables de escolaridad $s$ y de la información $q$. Para los períodos $\mathrm{x}>1$ los salarios se comportan de la siguiente manera:

$$
w_{x}=\lambda_{x}\left\{\left(\prod_{i=1}^{x}\left(1-\theta_{i}\right) \bar{z}+\left[1-\prod_{i=1}^{x}\left(1-\theta_{i}\right)\right] z\right\}+k_{x}\right.
$$

donde $k_{x}=\lambda_{x} \prod_{i=1}^{x}\left(1-\theta_{i}\right)\left[\alpha_{1} s+\alpha_{2}\left(\gamma_{1} s+v_{1}\right)\right]+c_{x}$.

Finalmente, se puede escribir la ecuación (vii) como:

$$
w_{x}=\lambda_{x}\left\{\left(1-\Theta_{x}\right) \bar{z}+\Theta_{x} z\right\}+k_{x}
$$

\section{REFERENCIAS}

Altonji, J. \& C. Pierret (1997). "Employer Learning and the Signaling Value of Education”. En I. Ohashi \& T. Tachibanaki (eds.), Industrial relations, incentives and employment. Londres: Macmillan. (2001). "Employer Learning and Statistical Discrimination". The Quarterly Journal of Economics, The MIT Press, 116(1): 313-50.

Arcidiacono, P., P. Bayer \& A. Hizmo (2010). "Beyond Signaling and Human Capital: Education and the Revelation of Ability". American Economic Journal: Applied Economics, 2(4): 76-104.

Bordón, P. \& B. Braga (2013). "Employer Learning, Statistical Discrimination and University Prestige" (manuscrito). University of WisconsinMadison.

Contreras, D., J. Rodríguez \& S. Urzúa (2013). "Sobre los orígenes de la desigualdad en Chile" (manuscrito). Universidad de Chile.

Farber, H. \& R. Gibbons (1996). "Learning and Wage Dynamics". The Quarterly Journal of Economics, The MIT Press, 111(4): 1007-47.

Lange, F. (2007). "The Speed of Employer Learning". Journal of Labor Economics, 25: 1-35.

Mansour, H. (2009). "Does Employer Learning Vary by Occupation" (manuscrito). University of California at Santa Barbara.

Ministry of Education (2012). Indicadores de educación.

Núñez, J. \& R. Gutiérrez (2004). "Discriminación social y meritocracia en el mercado del trabajo: el caso de Chile". Estudios de Economía, 31(2): 113-132.

Pasche, C. (2008). "A Multiple Ability Approach to Employer Learning" (mimeo). Universidad de Génova.

Repetto, A. \& R. Fischer (2003). "Método de selección y resultados académicos: Escuela de Ingeniería de la Universidad de Chile". Estudios Públicos 92 (primavera). 
Reyes L., J. Rodríguez \& S. Urzúa (2013). "Heterogeneous Economic Returns to Postsecondary Degrees: Evidence from Chile". Working Paper 18817, National Bureau of Economic Research.

Spence, M. (1973). "Job Market Signaling". The Quarterly Journal of Economics, The MIT Press, 87(3): 355-74.

Schönberg, U. (2007). "Testing for asymmetric employer learning”. Journal of Labor Economics, 25: 651-691.

Swett, J.P. (2011, April 4). CEO en Trabajando.com. Entrevista.

Weiss, A. (1995). "Human Capital vs. Signalling Explanations of Wages". Journal of Economics Perspectives, 9(4): 133-154. 